\title{
$K^{+} p$ ELASTIC SCATTERING AT 3.0 AND $3.5 \mathrm{GeV} / \mathrm{c}$
}

S. Debaisieux, F. Grard, S. Houghebaert, L. Pape, R. Windmolders

Laboratoire des Hautes Énergies, Brussels, Belgium

M. Ferro-Luzzi, R. George, Y. Goldschmidt-Clermont, V. P. Henri, B. Jongejans, D. W. G. Leith, G. R. Lynch, F. Muller, J.-M. Perreau

CERN, Switzerland

G. Otter, P. Sällström

Institute of Physics, Stockholm, Sweden

\section{THE PRODUCTION MECHANISM FOR THE REACTION $K^{+} p \rightarrow K^{*} p$ AT 3.0, 3.5 AND $5.0 \mathrm{GeV} / \mathrm{c}$}

M. Ferro-Luzzi, R. George, Y. Goldschmidt-Clermont, V. P. Henri, B. Jongejans, D. W. G. Leith, G. R. Lynch, F. Muller, J.-M. Perreau

CERN, Switzerland

\section{TWO-BODY PRODUCTION IN $K-p$ COLLISIONS AT $3 \mathrm{GeV} / \mathrm{c}$}

J. Badier, M. Demoulin, J. Goldberg, B. P. Gregory, P. Kresbich, C. Pelletier, M. Ville

Ecole Polytechnique, Paris, France

R. Barloutaud, A. Lèvéque, C. Louedec, S. Meyer, P. Schlein, A. Verglas

L. P C. H. E., Saclay, France

E. S. Gelsema, J. Hoogland, S. C. Kluyver, A. G. Tenner

University of Amsterdam, Holland

(Presented by Y. GOLDSCHMIDT-CLERMONT)

The present report concerns three exper1ments of $K^{+}$and $K^{-}$mesons performed at CERN in the Saclay $80 \mathrm{~cm}$ hydrogen bubble chamber.

I. The first experiment is a study of elastic scattering

$$
K^{+}+p \rightarrow K^{+}+p
$$

in a collaboration between Brussels, CERN and Stockholm. Preliminary results are presented below.

The angular distribution of the elastic scattering shows a dominant diffraction peak (Fig. 1). The angular distribution is fitted by an exponential, which corresponds to an optical model with black disc. The slope of the exponential is directly related to the disc radius.
The slope of the diffraction pattern is given as a function of $K^{+}$momentum on Fig. 2 . Our results are shown together with those obtained by other groups [1, 2]. A shrinking of the diffraction peak is observed, which is related to the increase of the inelastic cross section, while the total cross section remains constant.

II. The second experiment is a study of reaction

$$
K^{+}+p \rightarrow K^{*+}+p \rightarrow K^{0}+\pi^{+}+p
$$

by the CERN group.

Results are already published at $3 \mathrm{GeV} / \mathrm{c}$ $[3,4]$ Preliminary results are now presented for 3.5 and $5 \mathrm{GeV} / \mathrm{c}$. The study of the Dalitz plot (Figs. 3, 4 and 5) shows that 
the reaction $K^{+}+p \rightarrow K^{0}+p+\pi^{+}$proceeds through 3 channels

$$
\begin{gathered}
K^{+}+p \rightarrow K^{*+}+p \\
K^{+}+p \rightarrow K^{0}+K^{*++}+\pi^{+} . \\
\longrightarrow p+\pi^{+} .
\end{gathered}
$$

Non-resonant.

The results on reaction (1) are presented here. The $\Delta^{2}$-distribution shows a sharp peaking

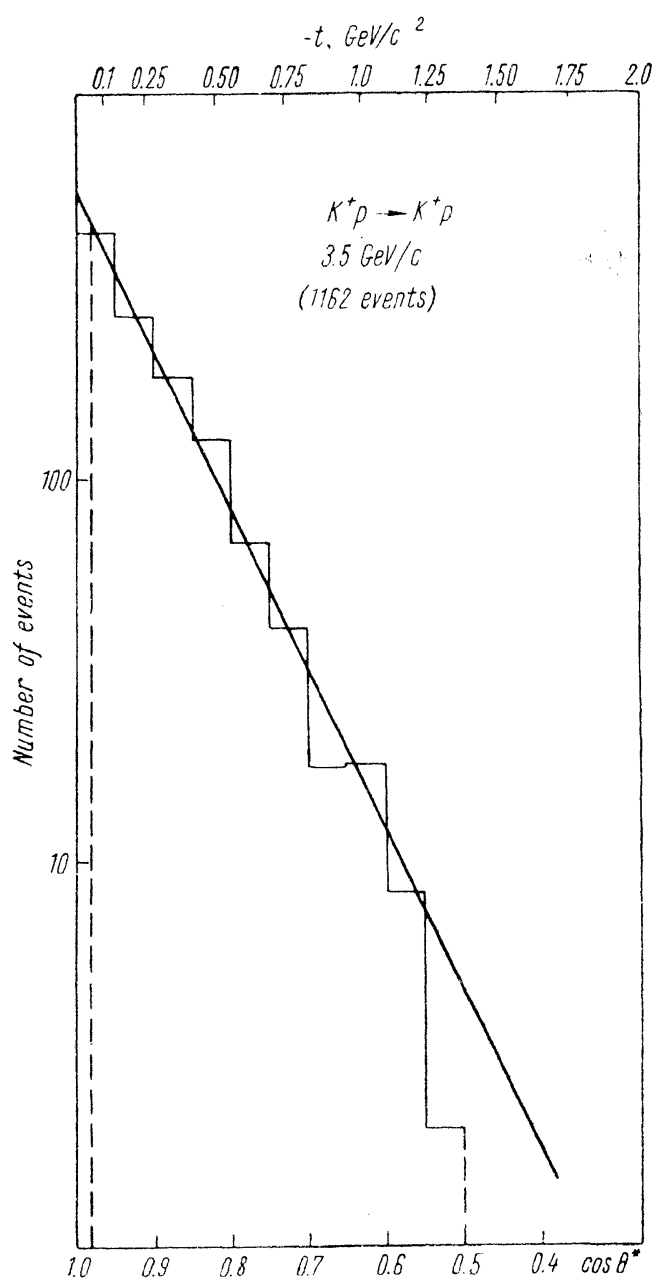

Fig. 1. The angular distribution for $K^{+} p$ elastic scattering at $3.5 \mathrm{GeV} / \mathrm{c}$. The distributions obtained at 3 and $5 \mathrm{GeV} / \mathrm{c}$ have a similar exponential behaviour.

characteristic of peripheral processes $[3,4]$. The reaction can thus be interpreted with the following graph (Fig. 6) and the analysis can be done with the assumption that the production mechanism is one-meson exchange.
The study of the angular distribution of the resonance decay gives information on the spinspace density matrix of the $K^{*}$ and on the exchanged particle.

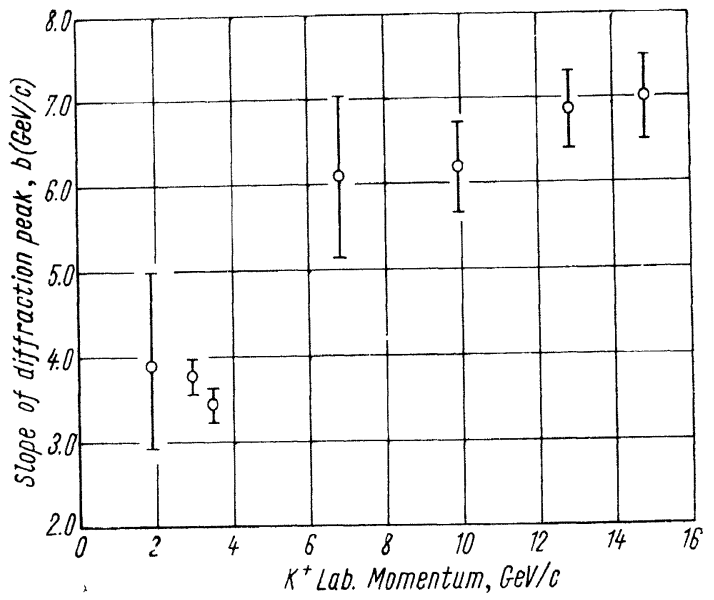

Fig. 2. The dependence of the slope of the elastic diffraction peak on incident momentum. The point at $2.0 \mathrm{GeV} / \mathrm{c}$ (Cook et al.) was wrongly plotted on the slide shown at the Conference. The points between (7-15) GeV, (Foley at al.), have been evaluated using a quadratic fitting for the cross section, and over a different range of $t$ values. If these latter points are treated on the same basis as our data, all the $K^{+} p$ scattering points fit a straight line $y=a x+b$ where $b=3(\mathrm{GeV} / \mathrm{c})^{-2}$ and $a=0.2(\mathrm{GeV} / \mathrm{c})^{-3}$.

If the axis of quantization is chosen along the direction of the incident $K^{+}$in the $K^{*}$ centre of mass system, it is easily shown that:

- with pion exchange the $K^{*}$ amplitude follows the spherical harmonic $Y_{1}^{0}$. The polar angular distribution is proportional to $\cos ^{2} \theta$ and the corresponding azimuthal distribution is flat. The $\varrho_{0}, 0$ matrix element is equal to 1 . - with vector meson exchange, the $K^{*}$ amplitude follows the spherical harmonics $Y_{1}^{1}$ and $Y_{1}^{-1}$. The polar angular distribution is proportional to $\sin ^{2} \theta$ and the azimuthal distribution is proportional to $\sin ^{2} \varphi$. Then $0_{0}, 0$ is equal to 0 Thus, the value of $Q_{0}, 0$ gives an estimate of the fraction of pion exchange in our simple one-meson exchange model.

At 3.5 and $5 \mathrm{GeV} / \mathrm{c}$ the $\theta$ and $\varphi$ angular distributions for the $K^{*}$ decay clearly show the dependence on $\sin ^{2} \theta$ and $\sin ^{2} \varphi$, indicating predominant vector meson exchange, as was already the case at $3 \mathrm{GeV} / \mathrm{c}[3,4]$.

Table summarizes the results obtained at three energies. It calls for the following comments:

- as the energy increases the $\Delta^{2}$ distribution shrinks and the parameter $\varrho_{0}, 0$ seems 


\begin{tabular}{l|c|c|c|c|c}
\hline & \multicolumn{3}{|c|}{$K^{+}$} & $K^{-}$ \\
\cline { 2 - 5 } & $\mathrm{GeV} / \mathrm{c}$ & 3 & 3.5 & 5 & 3 \\
\hline$\sigma_{t o t}$ & $\mathrm{mb}$ & $2.1 \pm 0.3$ & $2.2 \pm 0.3$ & $1.0 \pm 0.2$ & $1.49 \pm 0.3$ \\
$\sigma\left(K^{*} \rightarrow K^{0} \pi^{+}\right)$ & $\mathrm{mb}$ & $0.8 \pm 0.1$ & $0.8 \pm 0.1$ & $0.3 \pm 0.1$ & $0.76 \pm 0.1$ \\
$\Delta^{2}(60 \%$ of events $)$ & $(\mathrm{Gev} / \mathrm{c})^{2}$ & 0.5 & 0.4 & 0.24 & 0.5 \\
$\varrho_{0,0}$ & & $0.11 \pm 0.05$ & $0.21 \pm 0.08$ & $0.23 \pm 0.12$ & $0.17 \pm 0.05$ \\
$\varrho_{1,-1}\left(\varrho_{1,0}\right)$ & & $0.34 \pm 0.04$ & $0.18 \pm 0.08$ & $0.42 \pm 0.11$ & $0.29 \pm 0.04$ \\
& & $-0.05 \pm 0.04$ & & $-0.04 \pm 009$ & $0.01 \pm 0.02$ \\
\hline
\end{tabular}

to increase, which in our simple one-meson exchange model can be interpreted as an increase of pion exchange.

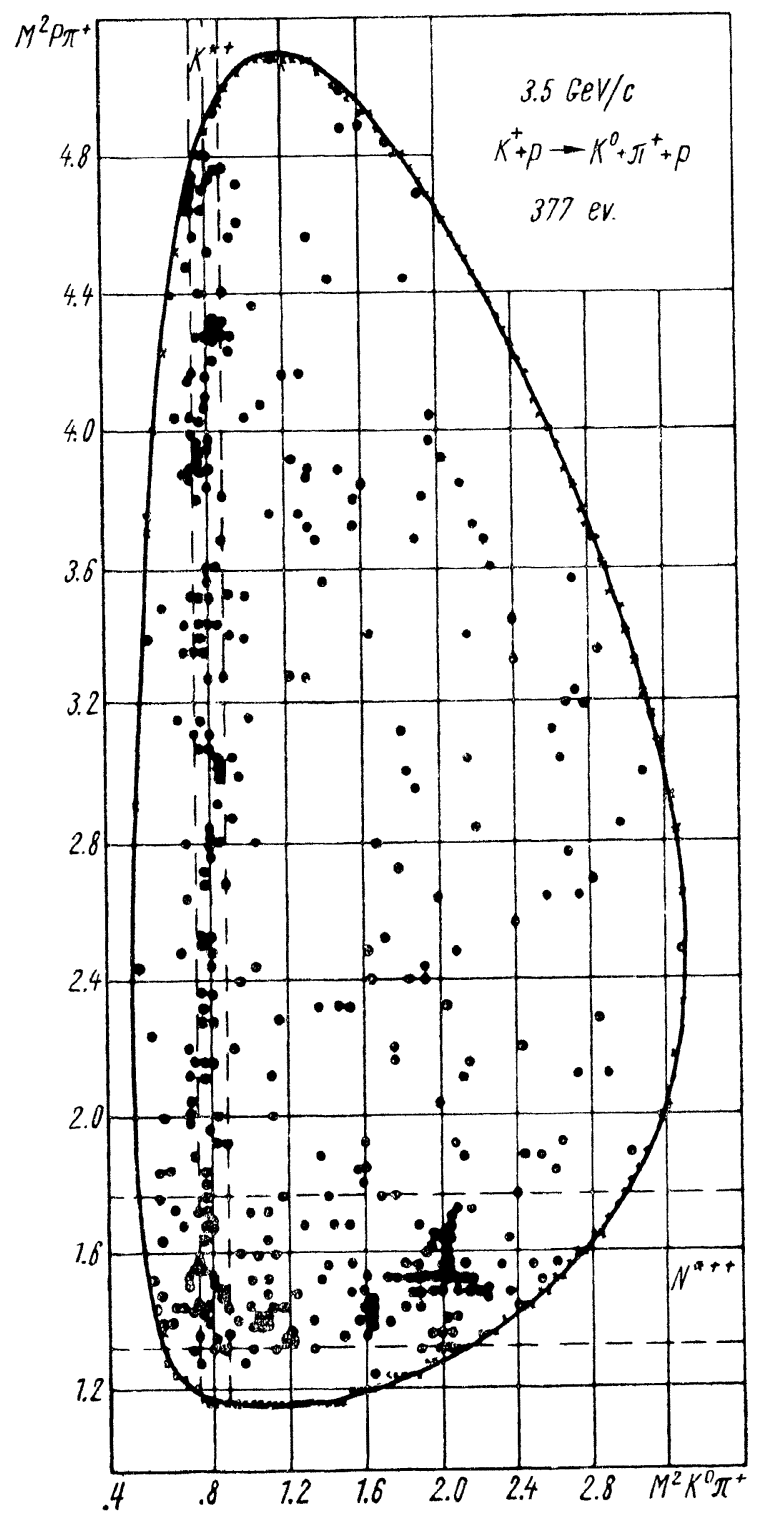

Fig. 3. Dalitz plot for the reaction $K^{+}+p \rightarrow K^{0}+$ $+\pi^{+}+p$ at $3.5 \mathrm{GeV} / \mathrm{c}$. The similar plots at 3 and $5 \mathrm{GeV} / \mathrm{c}$ show the same general behaviour.
However, Gottfried, Jackson and Svensson $[5,6]$ have developed a model taking into account the competition of absorptive processes. Using evidence from our elastic scattering results, they limit the partial-wave expansion of the propagator of the exchanged particle to large impact parameters. By adjusting the relative amounts of vector to pion exchange, as well as the ratio of vector to tensor coupling to fit the observed differential cross section, two solutions for the o parameters were obtained. One of them was in good agreement with the value of $\varrho_{0}, 0$ averaged over all production angles. This solution also predicted the variation of the $\varrho$ parameters as a function of $\Delta^{2}$. We have studied the $\Delta^{2}$ dependence of $\varrho_{0}, 0$, $\varrho_{1,-1}$ and $\operatorname{Re} \varrho_{1}, 0$ at $3 \mathrm{GeV} / \mathrm{c}$ and have found it to be in excellent agreement with the theoretical prediction given by the model [4].

III. The reaction

$$
K^{-}+p \rightarrow \dddot{K}^{0}+\pi^{-}+p
$$

was studied in a collaboration between Amsterdam, Excole Polytechnique an Saclay [7].

For the reaction

$$
\begin{aligned}
K^{-}+p \rightarrow \bar{K}^{*-}+p \\
\quad \longrightarrow \bar{K}^{0}+\pi^{+}
\end{aligned}
$$

the analysis was similar to that at CERN (moreover, a detailed moment analysis was made on the decay of the $\bar{K}^{*}$ and showed that the $\bar{K}^{*}$ can indeed be considered as a particle of spin 1, decaying freely). The results are given in Table.

It is seen that the spin-space matrix elements are in excellent agreement with the results for $K^{+}$. The $\Delta^{2}$ distribution is exactly the same as for the $K^{+}$. The results also show the $\Delta^{2}$ dependence of $\varrho_{0,0}$ and $\varrho_{1,-1}$, predicted by the Gottfried, Jackson and Svensson-model.

The $K^{-}$vertex is the exact charge conjugate of the $K^{+}$vertex. But the competitive absorp- 


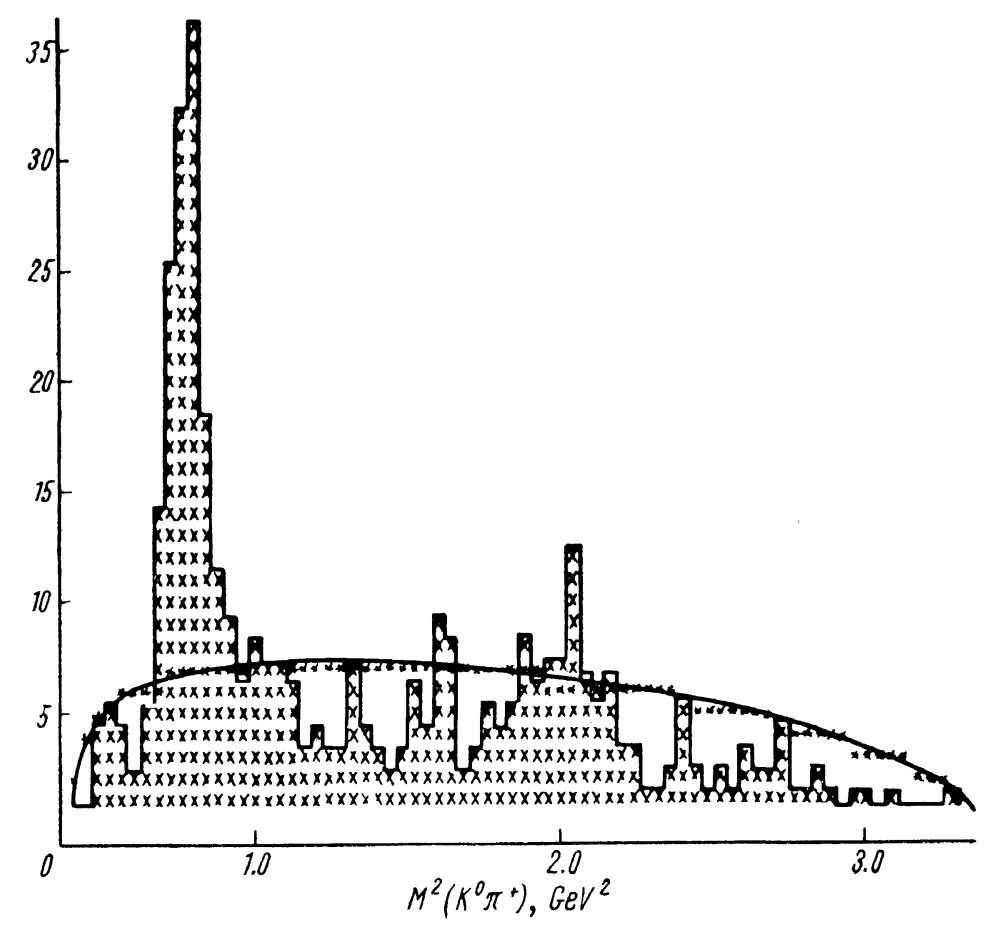

Fig. 4. The distribution of the $\left(K^{0} \pi^{+}\right)$effective mass squared, at $3.5 \mathrm{GeV} / \mathrm{c}$. The $K^{*+}$ resonance is clearly seen.

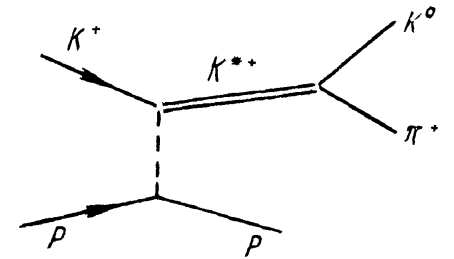

Fig. 6.

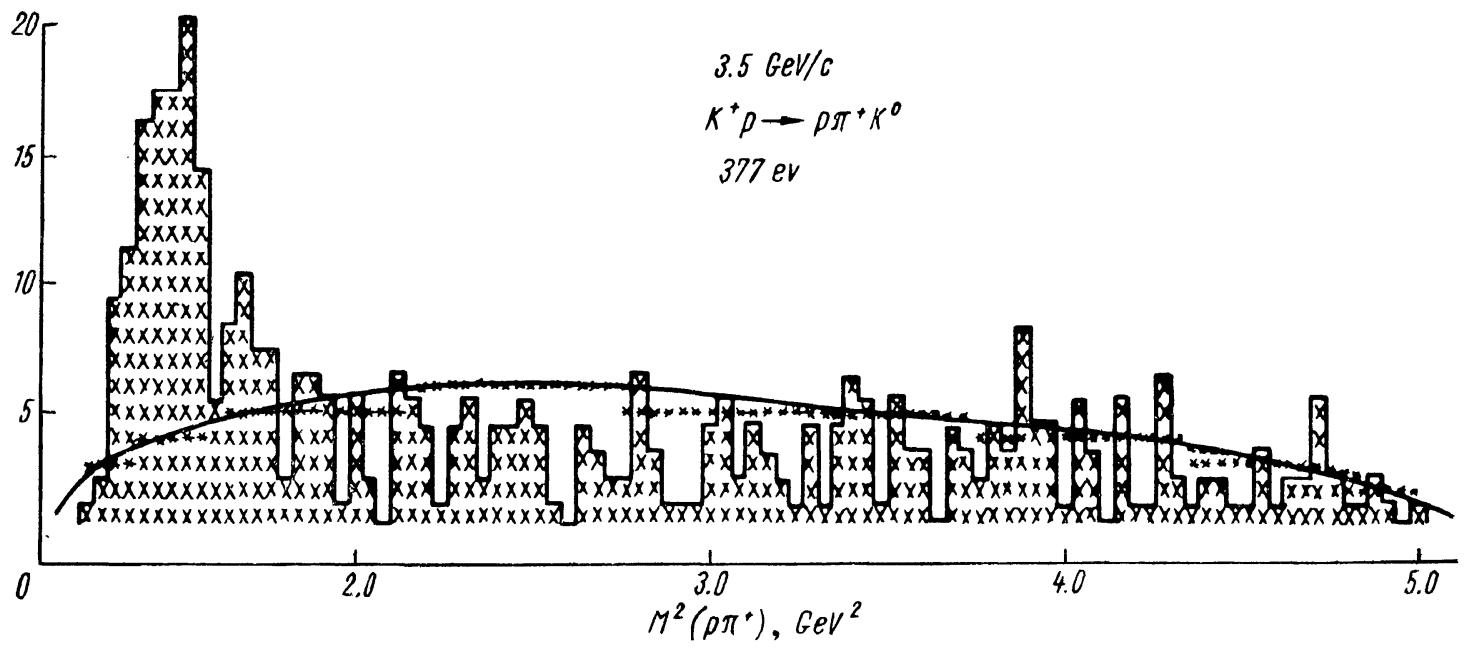

Fig. 5. The distribution of the $\left(p \pi^{+}\right)$effective mass squared, at $3.5 \mathrm{GeV} / \mathrm{c}$. The $N^{*++}$ resonance is clearly seeen.

tion processes are probably different in the two cases, which makes the apparent equality of the matrix elements more difficult to understand.

\section{REFERENCES}

1. Co o k V. et al. Phys. Rev,, 129, 2743 (1963). 2. F o 1 e y K. J. et al. Phys. Rev. Lett., 11, 503 (1963).

3. Ly n ch G. R. et al. Phys. Lett , 9, 359 (1964).
4. Ferro-Luzzi $M$. et al. Sent for publication.

5. Go t t f ried K., J a cks o n J. D. Phys. Lett., 8, 114 (1964); Nuovo cimento. 33, 309 (1964).

6. Got t f r i e d K. et al. "Influence of Absorption due to Competing Processes on Peripheral Reactions», Paper presented at the 1964 International Conference on High Energy Particles in Dubna.

7. B a di e r J. et al. To be published. 